\title{
Trace DNA evidence dynamics: An investigation into the deposition and persistence of directly- and indirectly-transferred DNA on
} reqularly-used knives

\author{
Georgina E. Meakin ${ }^{a, b *}$, Emma V. Butcher ${ }^{a, c}$, Roland A. H. van Oorschot ${ }^{d}$, Ruth M. \\ Morgan ${ }^{a, b}$ \\ a UCL Centre for the Forensic Sciences, 35 Tavistock Square, London, WC1H 9EZ, UK \\ ${ }^{b}$ UCL Department of Security and Crime Science, 35 Tavistock Square, London, WC1H 9EZ, UK \\ ' UCL Division of Biosciences, Medical Sciences Building, Gower Street, London, WC1E 6BT, UK \\ d Office of the Chief Forensic Scientist, Victoria Police Forensic Services Department, Melbourne, Australia \\ *Corresponding author: g.meakin@ucl.ac.uk
}

\section{Key words}

Forensic DNA analysis; trace DNA; transfer; persistence; regular use

\begin{abstract}
Empirical data on the transfer and persistence of trace DNA are crucial to the evaluation of forensic DNA evidence. This evaluation can be complicated by the occurrence of indirect DNA transfer; the possibility of which is well established, but research into such transfer is often focussed on unrealistic situations, e.g. handling of DNA-free items after participants have shaken hands for 1-2 minutes. To simulate more realistic scenarios, this study investigated the deposition and persistence of both directly- and indirectly-transferred DNA on knives that had been artificially set up as 'regularly-used'. Each knife was handled in a prescribed manner by a specific participant over two consecutive days to simulate regular use. Each participant then shook hands for 10 seconds with a fellow volunteer and immediately stabbed one of their knives into a foam block repeatedly for 60 seconds. DNA was recovered by minitaping from triplicate sets of knife handles from four pairings of volunteers after regular use, and at one hour, one day and one week after the handshaking and stabbing events.
\end{abstract}


Total amounts of DNA recovered from the knives, regularly used by a single person, varied among individuals; one volunteer consistently deposited significantly greater amounts than the others, whilst another volunteer did not always leave complete profiles. DNA attributed to the regular user persisted for at least a week, declining with increasing time between DNA deposition and recovery. Non-donor DNA was codeposited at $<5 \%$ of the profiles recovered, except for one volunteer, who consistently left DNA from their romantic partner on their knives at $\sim 25 \%$ and $\sim 11 \%$ of the profiles before and after the handshaking and stabbing events, respectively. In three pairings of volunteers, after the handshaking and stabbing events, alleles that could be attributed to the respective handshakers' profiles were detected as partial minor profiles, equating to $\sim 10 \%$ of the profiles recovered. For the fourth pairing of volunteers, only complete single-source DNA profiles matching the regular user's profile were recovered. However, it is important to note that, when indirectlytransferred handshaker DNA was detected, it declined with increasing time between DNA deposition and recovery.

These data provide an initial insight into the detection and persistence of directly- and indirectly-transferred DNA that extend the data already available on forensic DNA transfer. The results herein suggest that the sooner an item is sampled after an offence has occurred, the greater the chance of recovering indirectly-transferred DNA, which has implications for forensic reconstructions.

\section{Introduction}

Evaluation of trace evidence to assist a criminal investigation increasingly requires considerations at both the source and activity levels. Evaluation at the activity level ideally requires empirical data on the transfer and persistence of trace evidence in order to assess how the evidence was deposited at a crime scene, whether during the commission of a crime or through innocent means [1]. Of critical importance to this evaluation is a consideration of the possibility of indirect transfer. With regards to DNA as trace evidence, this is the transfer of DNA from a person to a surface of interest via an intermediary person(s) or object(s). Such indirect DNA transfer was initially 
identified in 1997 [2], and although there was some debate in the literature over the years that followed regarding the existence of indirect transfer and its relevance to casework, indirect transfer has been repeatedly demonstrated by empirical research and must be considered in the evaluation of trace DNA [3]. More recently, indirectlytransferred DNA has been observed in mock social settings [4] and stabbing simulations [5], and has been a key consideration in several high profile criminal cases, such as R v Reed \& Reed [6], Fitzgerald v The Queen [7] and the miscarriages of justice of Amanda Knox and Raffaele Sollecito [8]. Furthermore, given that DNA can persist on surfaces for a number of days or weeks depending on the environmental conditions [9] and on objects after use by a subsequent person [10-12], persistence of both directly and indirectly transferred DNA also needs to be considered in the evaluation of trace DNA in casework.

Indirect DNA transfer has commonly been investigated by asking volunteers to shake hands with a fellow participant and then hold or use a pre-cleaned item. Such experiments have yielded varied results, for example, DNA recovered from a plastic tube or glass beaker could be attributed to the handler and/or the handshaker, and the proportions of these DNA contributions varied from major handler to major handshaker $[13,14]$. However, the DNA profiling chemistries used in those studies have since been superseded by multiplexes with increased sensitivity. Interestingly, studies involving handshaking and handling experiments using these sensitive kits reported similarly varied results. For example, Cale et al. [15] found that 17 of 20 knives that yielded quantifiable DNA showed alleles matching those of the handshaker, with these alleles ranging from a minor profile to a major profile in the mixtures obtained. In particular, $25 \%$ of the profiles recovered showed a major component matching the profile of the handshaker [15]. Similarly, in a study involving handshaking and then handling of plastic tubes, $20 \%$ of the results obtained showed DNA from secondary transfer which exceeded that from primary transfer [16].

However, there are limitations to the value of these types of studies [17-19]. Two key drawbacks of these studies that limit their application to real-world casework situations are: the length of handshake used, which ranged from 30 seconds to 2 minutes [13- 
16], and the use of items that had been pre-cleaned to remove DNA. While a twominute handshake was specifically used in one study to mimic intimate contact [15], in general, such long time periods are not representative of everyday contact that one has with people in general, and it is this type of contact that is of particular interest when it comes to assessing the potential of innocently-transferred DNA to a crime scene. In addition, it is unlikely that surfaces are free of DNA before they come into contact with DNA from the offender or victim during a criminal act. Instead, a background layer of DNA is likely to already be present on surfaces, particularly of items that are regularly-used, such as items of clothing and some types of opportunistic weapons. This has recently been demonstrated when background DNA was detected on various public items, such as shopping baskets and stair rails, and private items, such as gloves and shirts [20].

It is therefore important to examine whether DNA indirectly-transferred via a handshake can still be detected on items when the contact time of the handshake is much shorter, such as just 10 seconds, and when the items have an initial background layer of DNA present. Using knives that were artificially set up as 'regularly-used', an initial study by Meakin et al. [21] showed that indirectly-transferred DNA from a 10 second handshake could indeed still be detected. It was also shown that the indirectlytransferred DNA could persist for at least a week and that DNA from other noncontrolled indirect DNA transfer events was detected. This paper extends these findings and the aims of Meakin et al. [21] by investigating the detection and persistence of DNA from the regular user on the knives and by providing a more thorough analysis of the indirectly-transferred DNA.

\section{Materials and Methods}

\subsection{Materials and volunteers}

Brand new steak knives with plastic handles (Cook Shop, UK) and $3 \mathrm{~L}$ and $4 \mathrm{~L}$ liddedplastic boxes (Office Depot, UK) were cleaned of any DNA present using MicroSol3+ or $10 \%$ bleach, followed by $70 \%$ ethanol and UV-irradiation for 20 minutes. Mini- 
tapes of three cleaned knife handles gave negative results when quantified and profiled for DNA, confirming this removal of DNA.

The knives were stored in batches in the $4 \mathrm{~L}$ plastic boxes, with the lids on to prevent contamination, until removed by a volunteer (see below). The $3 \mathrm{~L}$ boxes were used to construct stabbing apparatus, in which blocks of dry floral foam (Oasis, UK) were placed inside the boxes and covered in tinfoil (Fig. 1 (a)). The foil was cleaned with $10 \%$ bleach to remove any DNA present and replaced, along with the foam blocks, after each stabbing session.

Volunteers gave informed consent to participate in this study and four volunteers were selected (one male and three females, all Caucasian) who were available to attend the laboratory at the times required. Buccal swabs were taken from each volunteer, and from the romantic partner of one of the volunteers, to provide reference DNA samples. The volunteers were denoted $\mathrm{W}, \mathrm{X}, \mathrm{Y}$ and $\mathrm{Z}$, and the partner as $\mathrm{XP}$.

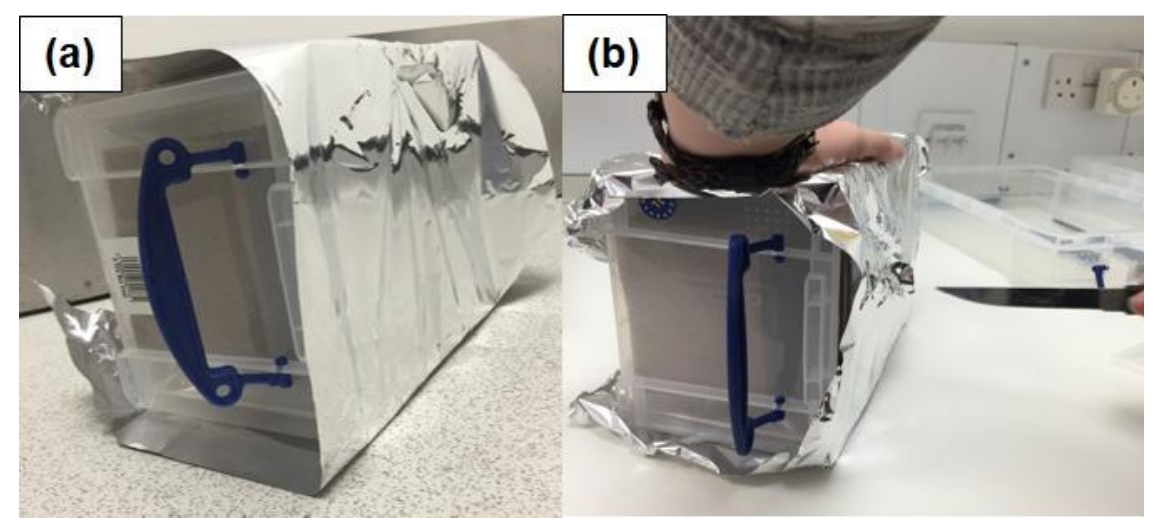

Fig. 1. The stabbing apparatus set-up (a) and the apparatus being stabbed by a volunteer (b).

\subsection{Experimental set-up}

Prior to laboratory visits, volunteers were instructed not to use any hand creams or anti-bacterial gels and not to wash their hands for at least one hour. They were also asked to avoid contact with the other volunteers taking part and touching any shared objects for the full duration of the study. 
To create a set of 'regularly-used' knives, volunteers attended the laboratory twice a day, with 6-7 hours between visits, for two consecutive days. They handled each of their three allocated knife handles per session with each hand for 30 seconds, comprising 15 seconds of holding and 15 seconds of rubbing, totalling four minutes of handling per knife over the two days. For the indirect transfer experiments, one set of three regularly-used knives per volunteer was set up each week for three consecutive weeks, giving a total of 36 knives. Twelve additional regularly-handled knives, three for each volunteer, were retained and mini-taped to provide DNA samples as positive controls.

Volunteers then attended the laboratory, in pairs, for three consecutive days with $\sim 24$ hours between each visit to give three replicates for each time point of sample collection. They shook hands for 10 seconds using their dominant hands, which for all the volunteers was their right hand. The length of the handshake was selected to maximise the likelihood of DNA transfer, whilst still being of a length that is realistic. Handshake lengths were measured from videos of handshakes between 30 different pairings of individuals giving a mean of $4.4 \pm 2.3$ seconds. The mean length plus two times the standard deviation was used (rounded up to 10 seconds), in order to include $97.5 \%$ of values drawn from a normal distribution.

Immediately following the handshake, and without touching anything else, each volunteer picked up a knife from their own set of 'regularly-handled' knives and proceeded to stab the stabbing apparatus repeatedly for 60 seconds (Fig. 1 (b)). To ensure consistency among volunteers and replicates, a metronome was used such that a rate of 1 stab every 2 seconds was maintained by all the volunteers, and directions were given to ensure volunteers each used a similar amount of force. A different knife was used each day for the stabbing to give triplicate results. The volunteer directly handling the knives is referred to as the 'regular user' and the volunteer they shook hands with as the 'handshaker'. 
DNA was recovered from the knife handles by using one mini-tape (WA Products Ltd., UK) per handle, with multiple applications of the tape repeatedly over the entire surface of the handle. For the experimental samples, mini-taping was performed at approximately one hour, one day, and one week after the handshaking and stabbing events. Volunteers therefore attended the laboratory on five consecutive days per week for three weeks to create samples for collection and examination of the DNA present at those three time points in triplicate.

\subsection{Processing of DNA samples}

DNA was extracted from the mini-tapes using the QIAamp® DNA Investigator Kit (QIAgen, Germany), as per the manufacturer's instructions for extracting DNA from cotton swabs. The mini-tapes were cut up into thin strips using DNA-free scissors prior to the addition of lysis buffers, and $1 \mu \mathrm{g}$ carrier RNA was added to improve the DNA extraction process. Thirty-five $\mu \mathrm{l}$ of Buffer ATE was used in the final elution and the resulting extracts were then stored at $-18^{\circ} \mathrm{C}$ until quantified and profiled. DNA was extracted from the buccal swabs using the SwabSolution ${ }^{\text {TM }}$ Kit (Promega, USA), as per the manufacturer's instructions.

DNA samples from the mini-tapes were quantified using the Quantifiler® Human DNA Quantification Kit (Applied Biosystems, USA) with the ABI PRISM® 7900HT Sequence Detection System (Applied Biosystems, USA), as per the manufacturer's instructions. DNA profiles were then obtained from $10 \mu$ l of each extract using the AmpF/STR $\circledast$ NGM SElect ${ }^{T M}$ PCR Amplification Kit (Applied Biosystems, USA), following the 30cycle PCR protocol, with the DNA Analyzer 3730xI (Applied Biosystems, USA) and analysed with GeneMapper $\AA^{8} 4.0$ software, all as per the manufacturer's instructions or the internal validation study of the laboratory. The DNA profiles obtained were interpreted, and a peak height threshold of 100 relative fluorescence units (rfu) used, in accordance with the laboratory's internal validation study. 


\subsection{Data analyses}

The reference profiles generated from the buccal swabs were used to determine the relative contributions of the regular user and handshaker to the profiles obtained from the knife handles. These values were calculated using the relative peak height contributions from the unique alleles that could be attributed to each of the respective reference profiles at each locus, with the exclusion of the Amelogenin locus. The relative profile contributions were averaged across all sixteen STR loci and across the three replicates per sample. Trends in or differences between data sets were

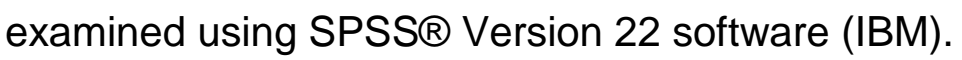

To attribute approximate evidential weights to the comparison of the DNA profiles from the knives handled by volunteer $X$ to the reference profiles of $X$ and $X P$, both a random match probability (RMP) and a range of likelihood ratios (LRs) were determined. The RMP was calculated manually and the LRs were determined using LRmix Studio 2.0 as per the developer's instructions [22]. These calculations used UK Caucasian allele frequencies and an FST value of 0.03 , as per directions from the UK's Forensic Science Regulator's guidance [23].

\section{Results}

\subsection{Transfer of DNA to knife handles during 'regular use'}

To maintain an element of control in the DNA deposited on the knives during regular use, the knives were first cleaned to remove all DNA; the handles of three such knives were mini-taped and no DNA was detected by both quantification and profiling. Cleaned knives were then handled in a prescribed manner across two days and three regularly-used knives from each of the four volunteers were mini-taped to examine the quantities of DNA and the nature of the DNA profiles initially deposited, prior to the handshaking and stabbing stage of the experiment. As reported previously, the average total quantities of DNA recovered from these knife handles were $3.4 \pm 0.5$, $0.9 \pm 0.8,1.2 \pm 0.5$ and $10.4 \pm 3.7 \mathrm{ng}$ for volunteers $\mathrm{W}, \mathrm{X}, \mathrm{Y}$ and $\mathrm{Z}$, respectively [21] Using an ANOVA test, it was found that the amounts of DNA deposited on the knives during regular use significantly differed among the volunteers $(F=30.79, p<0.01)$. To 
examine this further, individual pairwise tests using the Student's t-test were conducted. These found that volunteer $Z$ had deposited significantly more DNA than the other volunteers, volunteer $\mathrm{W}$ had deposited significantly more DNA than volunteers $X$ and $Y$, and that the amounts of DNA deposited by volunteers $X$ and $Y$ were not significantly different $(p<0.01$ for all comparisons). With respect to intravolunteer variation, the variation within the samples for each individual was less than $42 \%$ for volunteers $\mathrm{W}, \mathrm{Y}$ and $\mathrm{Z}$, but much higher for volunteer $\mathrm{X}$ at $89 \%$ (Fig. 2).

All of these knife handles gave major profiles of the regular user, with those from volunteers $\mathrm{W}, \mathrm{Y}$ and $\mathrm{Z}$ giving complete profiles. Some drop-out of alleles from the

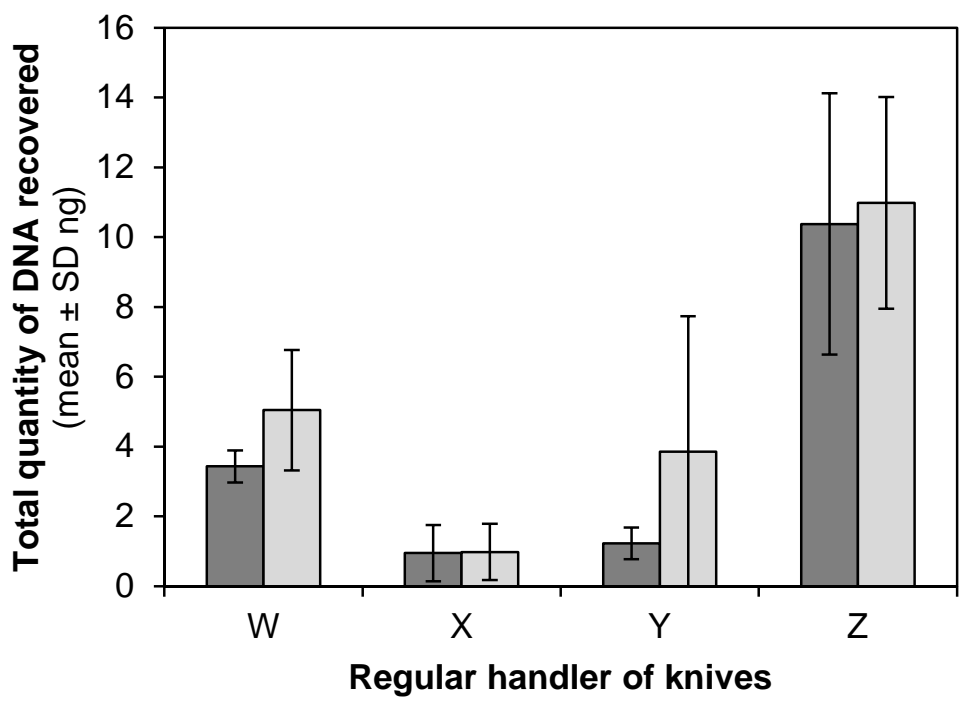

Fig. 2. DNA recovered from regularly-used knife handles before (dark grey bars) and one hour after (light grey bars) users shook hands with a fellow volunteer and stabbed the knives into a foam block. Quantities of DNA are presented as means of three replicate knives \pm one standard deviation (SD).

regular user was observed for volunteer X's knives, such that these knives gave partial profiles of $77-93 \%$. The regularly-used knife handles (prior to handling after handshake) from all the volunteers also showed additional alleles that could not have come from the regular user, observed as minor profiles (Table 1). Based on peak heights and number of alleles, these non-donor alleles comprised $1-3 \%$ of the profiles for volunteers $\mathrm{W}, \mathrm{Y}$ and $\mathrm{Z}$, but $\sim 25 \%$ of the profiles for volunteer $\mathrm{X}$; a representative 
profile from volunteer X's knives is shown in Fig. 3. Treating this profile as a crimestain profile with a known source, subtracting the alleles from the known source (volunteer X) gave partial DNA profiles for each of the three replicates; alleles that were detected in more than one replicate sample were then included in a consensus profile (Table 1). In an attempt to identify the possible source of this consensus DNA profile, it was compared to profiles from users of the laboratory and volunteers in other studies, but no match was observed. On speaking with volunteer $X$, they suggested their partner as a potential source and he subsequently provided a buccal swab to generate a reference DNA profile (XP). This profile was found to match the consensus profile with a RMP of 1 in 94,000 . 


\begin{tabular}{|c|c|c|c|c|c|c|c|c|c|c|c|c|c|c|c|c|c|c|}
\hline Regular user & Replicate & D10S1248 & vWA & D16S539 & D2S1338 & Amel & D8S1179 & D21S11 & D18S51 & D22S1045 & D19S433 & TH01 & FGA & D2S441 & D3S1358 & D1S1656 & D12S391 & SE33 \\
\hline \multirow{3}{*}{ W } & 1 & - & - & - & $25-$ & - & 91011 & $28-$ & - & $16-$ & - & $7-$ & - & - & - & - & - & - \\
\hline & 2 & $13-$ & - & - & - & - & $9-$ & - & $14-$ & - & - & 79.3 & - & - & $17-$ & - & - & $30.2-$ \\
\hline & 3 & - & - & - & - & - & - & $28-$ & - & - & - & - & - & 1011 & $14-$ & - & - & $30.2-$ \\
\hline \multirow{3}{*}{$Y$} & 1 & $14-$ & - & - & - & - & $12-$ & - & - & 1116 & - & - & - & $11-$ & - & - & - & - \\
\hline & 2 & $14-$ & - & - & - & - & - & - & - & $16-$ & - & - & - & - & - & - & - & - \\
\hline & 3 & $14-$ & - & - & - & - & - & - & - & $16-$ & - & - & - & - & - & - & - & - \\
\hline \multirow{3}{*}{ z } & 1 & - & - & - & - & - & 131415 & 30 & - & - & - & - & - & $11-$ & - & - & - & - \\
\hline & 2 & - & - & - & - & $Y$ & 1314 & 2934.2 & - & - & - & - & - & 1011 & - & - & - & - \\
\hline & 3 & - & 1516 & - & - & - & 1416 & 2931 & - & $16-$ & $16-$ & $9.3-$ & - & 1011 & $14-$ & - & - & - \\
\hline \multirow{6}{*}{$x$} & 1 & $13-$ & - & - & - & $Y$ & $15-$ & $31-$ & - & $16-$ & 1314 & $9-$ & $23-$ & - & $15-$ & - & - & - \\
\hline & 2 & 1315 & - & - & - & $Y$ & $15-$ & $31-$ & - & $16-$ & 1314 & $9-$ & - & $11-$ & $15-$ & - & $16-$ & - \\
\hline & 3 & 1315 & - & - & - & $Y$ & $15-$ & $27-$ & - & $16-$ & 1314 & $9-$ & 2023 & $11-$ & $15-$ & - & - & - \\
\hline & Consensus & 1315 & - & - & - & $Y$ & $15-$ & $31-$ & - & $16-$ & 1314 & $9-$ & $23-$ & $11-$ & $15-$ & - & - & - \\
\hline & XP ref & 1315 & 1518 & 1213 & 1723 & $X Y$ & 1215 & 2731 & 1720 & 1617 & 1314 & 9 & 2023 & 1011 & 1516 & 1516 & 1617 & 26.228 .2 \\
\hline & $Z$ ref & 1315 & 1718 & 12 & 1722 & $x x$ & 911 & 33.2 & 1317 & 1115 & 1414.2 & 69 & 2326 & 14 & 16 & 1315.3 & 1519 & 1420 \\
\hline
\end{tabular}

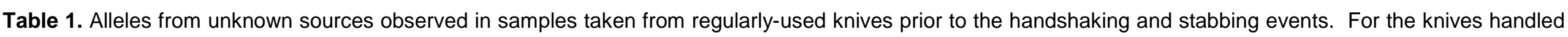

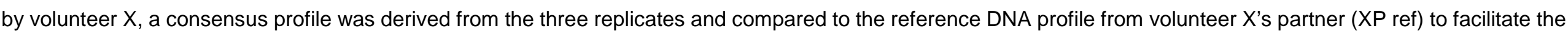

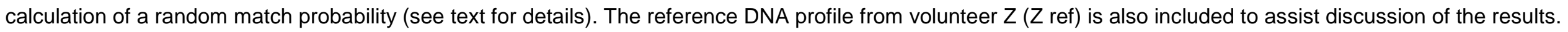


(a)

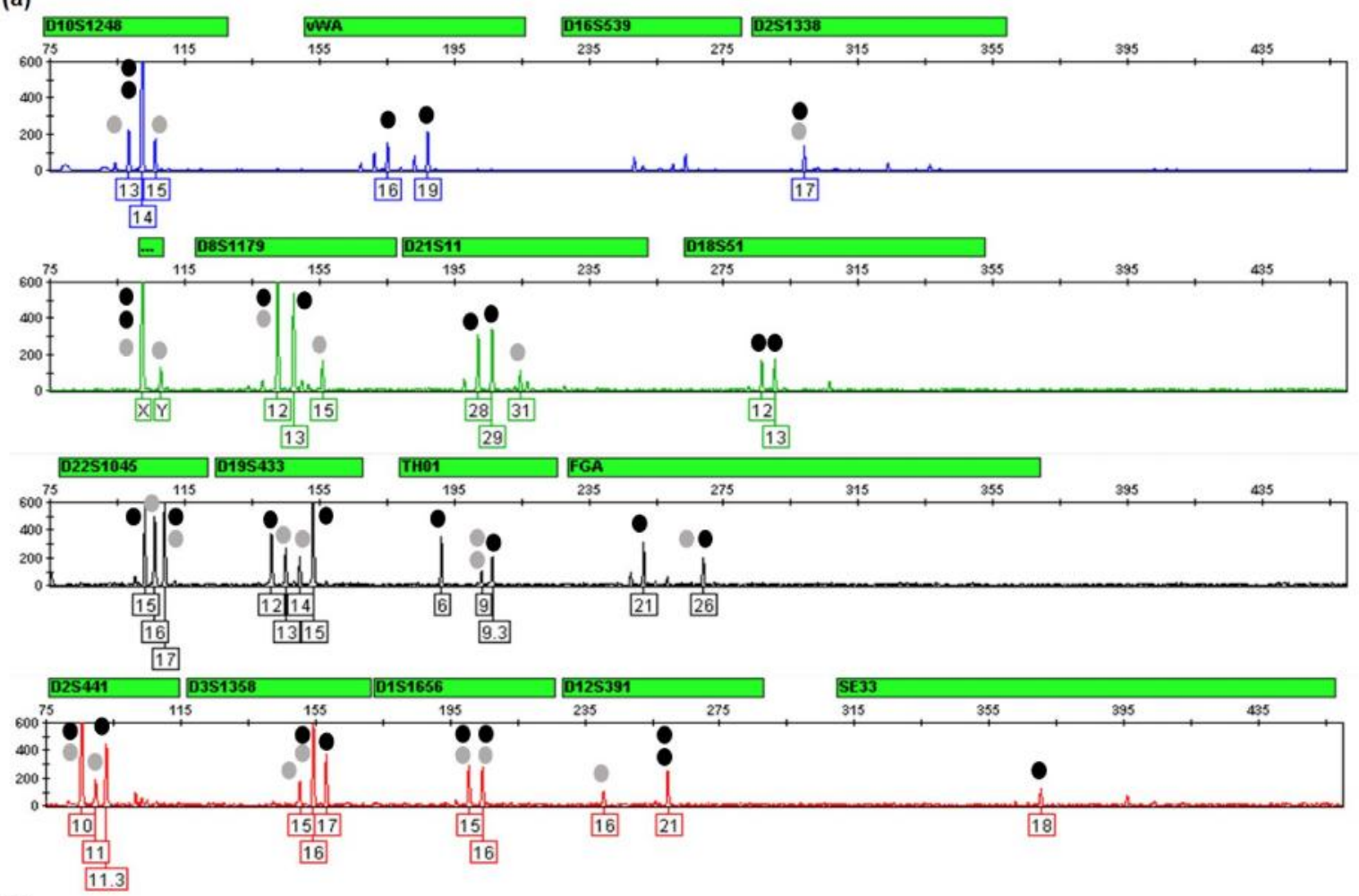

(b)

\begin{tabular}{|l|l|l|l|l|l|l|l|l|l|l|l|l|l|l|l|l|l|}
\hline Reference & D10S1248 & WWA & D16S539 & D2S1338 & Amel & D8S1179 & D21S11 & D18S51 & D22S1045 & D19S433 & TH01 & FGA & D2S441 & D3S1358 & D1S1656 & D12S391 & SE33 \\
\hline
\end{tabular}

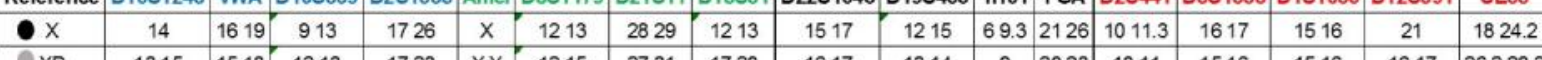

Fig. 3. An example epg of the DNA samples taken from the knives regularly-used by volunteer $X$ (a) with the DNA profiles (b) of volunteer $\mathrm{X}(\mathrm{X})$ and their partner $(\mathrm{XP})$.

However, in recent years, casework laboratories are tending to use statistical software to determine likelihood ratios instead of calculating RMPs, since software programmes can take into account shared alleles and replicate analyses. As such, instead of subtracting the alleles attributed to volunteer $X$ and generating a resultant consensus profile, all three replicate samples were entered into the LRmix Studio software, along with $X$ and $X P$ reference profiles for comparison. Two hypotheses were then compared: that the sample profiles could be explained by contributions from $X$ and XP $\left(H_{p}\right)$ versus by contributions from $X$ and an unknown $\left(H_{d}\right)$. Given the absence of contamination in the negative controls and extraction blanks processed during this 
study, it was considered that no drop-in had occurred, and therefore a probability of 0 was selected for drop-in. Average drop-out probabilities across the three replicate samples of 0.14 and 0.44 were determined under $H_{p}$ for the contributions made by $X$ and XP, respectively. Use of the sensitivity analysis module of LRmix Studio gave a range of drop-out probabilities for the unknown under $\mathrm{H}_{d}$ of 0.33 to 0.76 . Using these probabilities, a range of LRs were determined from 542,000 to $4.86 \times 10^{9}$ (to 3 significant figures).

\subsection{Persistence of total DNA on 'regularly-used' knife handles}

For the remaining 'regularly-used' knives, the volunteers shook hands for 10 seconds with a fellow volunteer and then took one of their own knives and stabbed it into a foam block for 1 minute. For each pair of volunteers, this was repeated to give three sets of three knives that had been stabbed into foam following a handshake and DNA was recovered from a set of knives at one hour, one week and one day following the stabbings. When DNA was recovered one hour after the stabbings, the total amount of DNA recovered was not significantly greater than that recovered prior to the additional handling of the knives (Fig. 2).

Analysis of the mini-tapes from the knives at one day and one week after the stabbings showed that DNA persisted on the knife handles for at least a week (Fig. 4). Comparison of the total quantities of DNA persisting on the knife handles across the one-week period showed that there was a gradual decline in the quantities of DNA recovered for knives handled by volunteers $\mathrm{W}, \mathrm{X}$ and $\mathrm{Y}$, although there was an unexpected increase in the amount of DNA from volunteer Z's knives at one day after the stabbings, before it too declined at one week (Fig. 4). A Pearson's correlation was used to explore whether the total DNA quantity recovered was significantly related to time between DNA deposition and recovery for each of the volunteers' sets of knives. For volunteers $\mathrm{W}$ and $\mathrm{Z}$, there were statistically significant very strong $(r=-0.88$, $p<0.01)$ and moderate $(r=-0.51, p<0.05)$ negative correlations between total DNA recovery and time since DNA deposition, respectively. However, for volunteers $X$ and $Y$, whilst a weak negative correlation was observed ( $r=-0.39$ for both), this was not statistically significant $(p=0.1)$. 


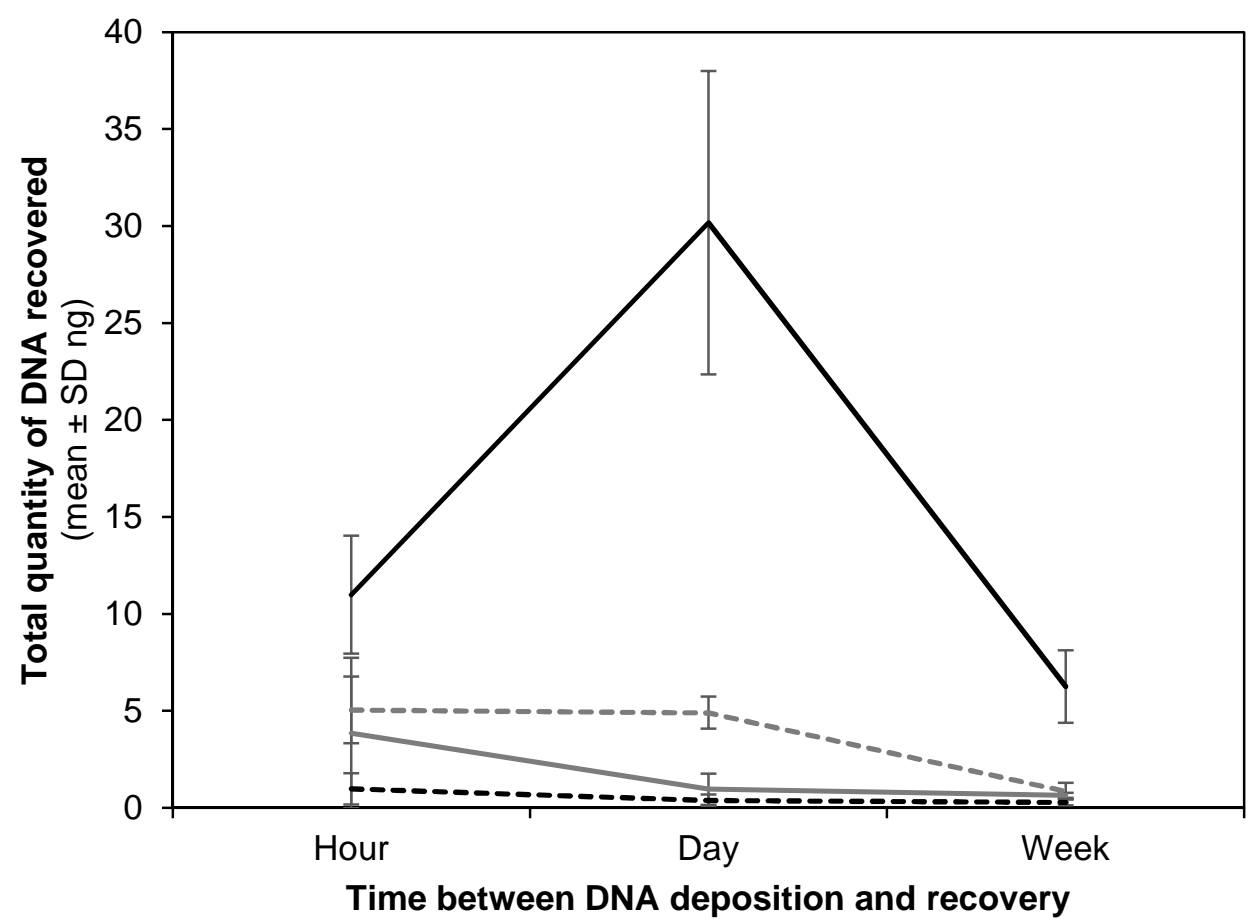

Fig. 4. Quantities of DNA recovered from regularly-used knife handles at approximately one hour, one day and one week after the regular user shook hands with a fellow volunteer and stabbed the knives into a foam block. Knives were handled by volunteer $W$ (grey dashed line), $X$ (black dashed line), $Y$ (grey solid line) and Z (black solid line). Quantities of DNA are presented as means of three replicate knives \pm one standard deviation (SD).

\subsection{Persistence of DNA from regular user on knife handles}

To gain a better understanding of the sources and persistence of the total DNA being deposited on the knives, the samples were profiled and DNA attributed to the regular users of the knives was initially examined. For volunteers $\mathrm{W}$ and $Z$, complete profiles matching those of the regular users were recovered from the knife handles at all time points (Fig. 5a). This corresponded with the observations that, when compared to the other two volunteers, these two volunteers deposited the greatest amounts of DNA (Fig. 2) with the highest allele peak heights (Fig. 5b). Over the course of the week, the profile percentage of DNA attributed to volunteer $X$ reduced from an average of $99 \%$ to an average of $83 \%$ (Fig. 5a). This also corresponded with observations of relatively low peak heights (Fig. 5b) and the low amounts of DNA deposited by this volunteer (Fig. 2). Relatively low amounts of DNA were also deposited by volunteer 
$Y$ (Fig. 2), which were reflected in the slight decline of average profile percentage from $100 \%$ to $99 \%$ across the week (Fig. $5 \mathrm{a}$ ) and the allele peaks being of heights between those for volunteer $X$ and those for volunteers $W$ and $Z$ (Fig. 5b). For all the volunteers, the peak heights of regular user alleles showed a decrease from one day after the stabbings to one week (Fig. 5b). This corresponded with the trends seen with the total amounts of DNA (Fig. 4), including an increase in peak height of volunteer Z's alleles at one day after the stabbings (Fig. 5b). A Pearson's correlation was used to explore whether the peak heights of regular user alleles were significantly related to time since DNA deposition. For volunteers $\mathrm{W}, \mathrm{X}$ and $\mathrm{Y}$, there were statistically significant moderate negative correlations between regular user peak heights and time since DNA deposition ( $r=-0.57,-0.49$ and -0.41 , respectively, $p<0.01)$. However, only a very weak negative correlation was observed between allele peak heights from volunteer $\mathrm{Z}$ and time since DNA deposition, and this was not statistically significant $(p=0.06)$. This is likely due to the greater amounts of DNA recovered from this volunteers' knives (Fig. 2). 

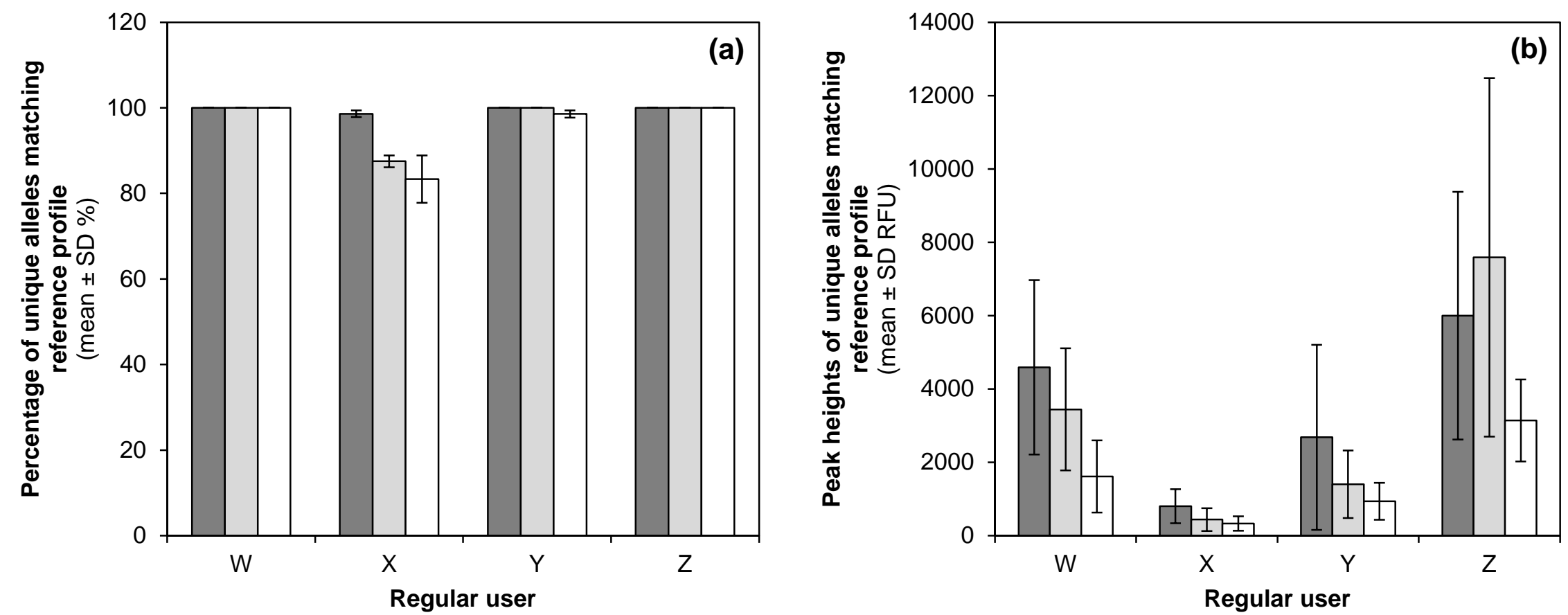

Fig. 5. Percentage of unique alleles (a) and their peak heights (b) matching the reference profiles of the respective regular users of the knives. DNA was recovered from regularly-used knife handles at approximately one hour (dark grey bars), one day (light grey bars) and one week (white bars) after the regular user shook hands with a fellow volunteer and stabbed the knives into a foam block. Percentages of alleles and peak heights are presented as means of three replicate knives \pm one standard deviation (SD). 


\subsection{Indirectly-transferred DNA to 'regularly-used' knife handles}

The DNA profiles from the knife handles were then examined for DNA from sources other than the regular user for each set of knives. Fig. 6 shows the relative contributions of DNA from the regular user, handshaker and unknown sources for DNA profiles recovered from the knife handles one hour after the handshaking and stabbing events. In three of the four pairings of volunteers, alleles that could be attributed to the respective handshakers' profiles were detected, along with alleles from unknown sources, but no non-donor alleles were detected for the Z:X pairing (Fig. 6). No DNA that could be attributed to indirect transfer from the handshaker (volunteer $X$ ) was detected on volunteer Z's knives. This was expected given the difference in the amounts of DNA deposited by these two individuals (Fig. 2). However, it was surprising that no unknown non-donor DNA was observed, given that unknown alleles had been detected on the regularly-used knives by volunteer $Z$, prior to the handshaking and stabbing events (Table 1).

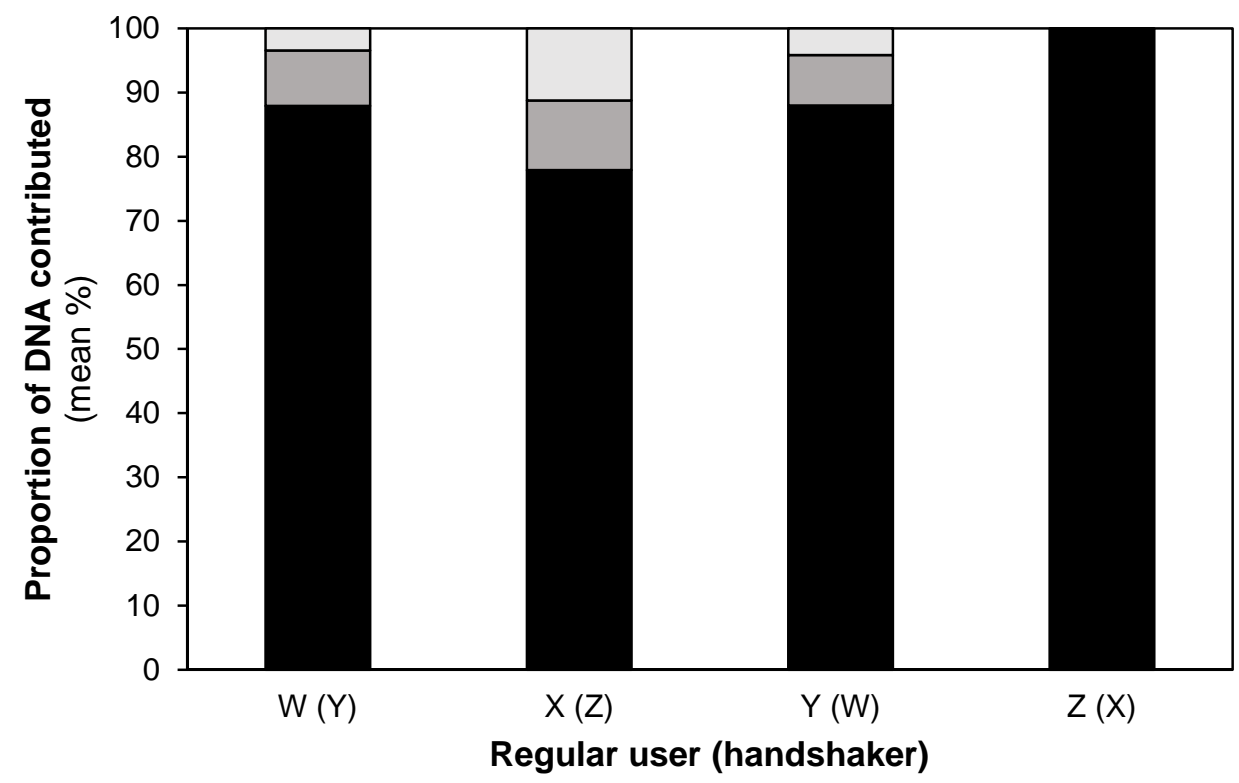

Fig. 6. Proportion of DNA contributed from the regular user (black bars), handshaker (dark grey bars) and other unknown sources (light grey bars) to the mixed DNA profiles recovered from regularly-used knife handles at approximately one hour after the regular user shook hands with a fellow volunteer and stabbed the knives into a foam block. 
With regards to the non-donor DNA in the other volunteer pairings, as reported previously, DNA that could be attributed to the respective handshakers' profiles were detected at ratios to regular user DNA of approximately 1:10, 1:7 and 1:11 for the pairings of volunteers $\mathrm{W}: Y, X: Z$ and $Y: W$, respectively [21], equating to approximately $10 \%$ of the total profiles for all three pairings (Fig. 6). DNA from unknown sources was also observed, comprising 3-4\% of the total profiles for the pairings of volunteers $W: Y$ and $\mathrm{Y}: \mathrm{W}$ (Fig. 6), which is a similar level to the unknown DNA observed in the profiles obtained from these volunteers' knives before the handshaking and stabbing events. However, for the $X: Z$ pairing, $11 \%$ of the total profiles obtained could not be attributed to the regular user (volunteer $\mathrm{X}$ ) or the handshaker (volunteer $\mathrm{Z}$ ). These alleles were the same as those non-donor alleles observed prior to the handshaking and stabbing events (Table 1) and were therefore also attributed to the DNA profile of volunteer X's partner (Fig. 3).

\subsection{Persistence of indirectly-transferred 'handshaker' DNA on knife handles}

To examine the persistence of indirectly-transferred DNA on the knife handles, only the alleles that could be attributed to the respective handshakers' profiles were considered. DNA attributed to the handshakers could still be detected at a week after the handshaking and stabbing events (Fig.7), demonstrating that indirectlytransferred DNA can persist for at least a week. However, these alleles were detected as minor profiles and no full profiles of unique alleles attributed to the handshakers' profiles were observed in any of the pairings of volunteers (Fig. 7a). For the pairings of volunteers $\mathrm{W}: \mathrm{Y}$ and $\mathrm{Y}: \mathrm{W}$, a decline in the profile percentages of the handshakers' DNA was observed with increasing time since DNA deposition on the knife handles (Fig. 7a). For $\mathrm{W}: \mathrm{Y}$, this was a statistically significant very strong negative correlation (Pearson's $r=-0.87, p<0.01$ ), whereas for $\mathrm{Y}: \mathrm{W}$, this was a weak negative correlation that was not statistically significant (Pearson's $r=-0.34, p=0.38$ ). For the pairing of $X: Z$, the profile percentage of handshaker DNA unexpectedly increased with increasing time since DNA deposition (Fig. 7a), although this was not a statistically significant correlation (Pearson's $r=0.52, p=0.15$ ). 

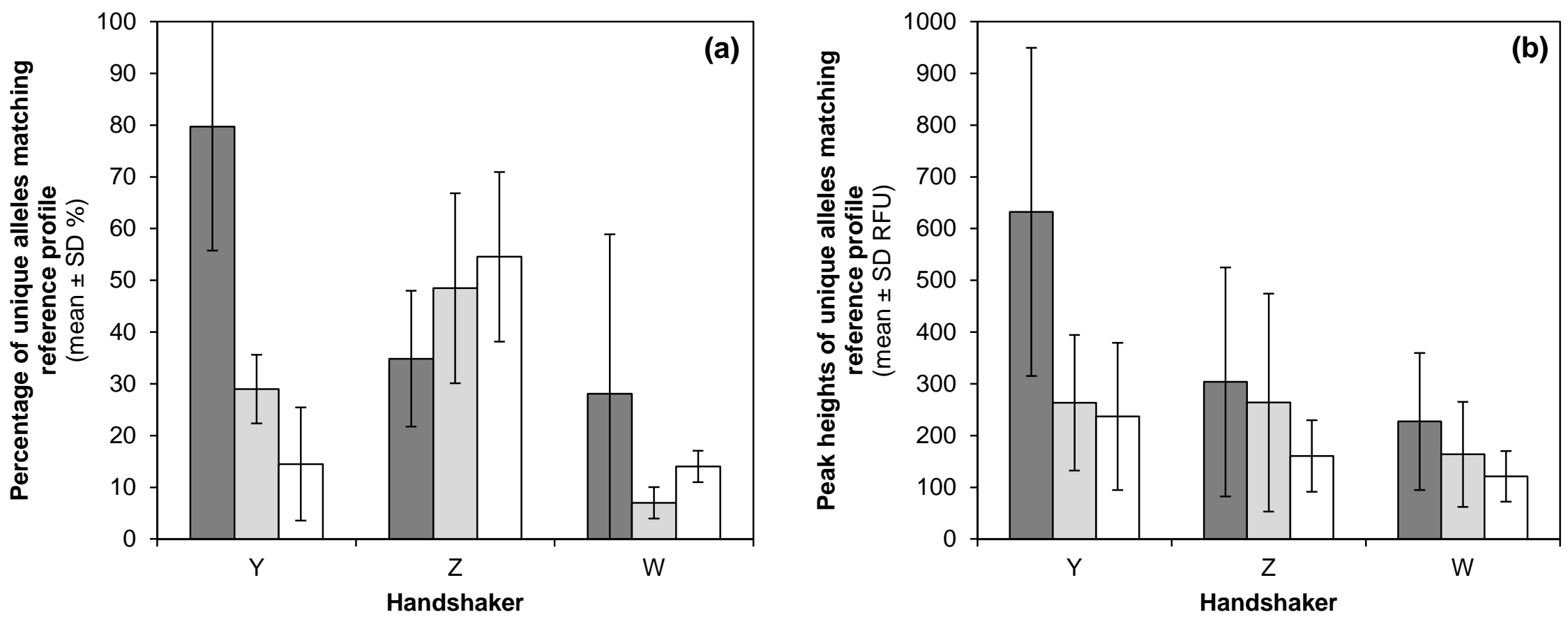

Fig. 7. Percentage of unique alleles (a) and their peak heights (b) matching the reference profiles of the respective volunteers that shook hands with the regular users of the knives. DNA was recovered from regularly-used knife handles at approximately one hour (dark grey bars), one day (light grey bars) and one week (white bars) after the regular user shook hands with the fellow volunteer and stabbed the knives into a foam block. Percentages of alleles and peak heights are presented as means of three replicate knives \pm one standard deviation (SD). 
However, in line with the observations of the regular user DNA, the peak heights of handshaker alleles significantly reduced with increasing time since DNA deposition for all the pairings of volunteers (Fig. 7b). This was a moderate correlation for the pairings of $\mathrm{W}: \mathrm{Y}$ and $\mathrm{Y}: \mathrm{W}$ (Pearson's $r=-0.55, p<0.01$ and $r=-0.42, p<0.05$, respectively) and a weak correlation for X:Z (Pearson's $r=-0.32, p<0.01$ ).

\section{Discussion}

The results of this study show that regular handling of knives gave detectable levels of regular user DNA that persisted for at least a week, varied between individuals, and did not always result in complete DNA profiles. Given this background layer of DNA, the results expanded herein and first reported by Meakin et al. [21] also show that when knife handles had a background level of DNA present, indirectly-transferred DNA could still be detected and such indirectly-transferred DNA could persist for at least a week. However, the indirectly-transferred DNA was only observed as minor partial profiles, which reduced with increasing time between DNA deposition and recovery, suggesting that the sooner an item is sampled after an offence has occurred, the greater the chance of recovering indirectly-transferred DNA.

\subsection{Deposition of regular user DNA}

The amounts of DNA recovered from the knife handles, which had been handled in a prescribed manner to simulate regular use, were in the range of approximately 1$10 \mathrm{ng}$, a range that is comparable to those amounts previously reported for actual regularly-used items of a similar material (plastic), such as pens, car keys, lighters, lipsticks and sunglasses [2,12]. Although variation was recorded among the amounts of DNA deposited by a single volunteer and among the amounts of DNA deposited between individuals, as has been previously observed (for example, see [16] and [5]), there appeared to be more variation between individuals than between samples from the same individual.

Whilst this appears to support the concept of 'shedder-status' as initially proposed by Lowe et al. [14], especially given the stark contrast between the amounts of DNA 
consistently deposited by volunteers $X$ and $Z$ (Fig. 2), it has been proposed that the ability of a person to shed DNA can vary depending on, for example, the time of day and the activities conducted immediately prior to DNA deposition [24]. To try to mitigate these factors, attempts were made to ensure that volunteers attended the laboratory at the same time of day each day and did not to use any hand creams or anti-bacterial gels or wash their hands for at least one hour prior to participating. This may therefore explain why volunteers in this study appeared to deposit amounts of DNA that consistently differed among the participants. The observation that volunteer $Z$ consistently deposited greater amounts of DNA than the other volunteers is also supported by a recent investigation into shedder status; it reported that some individuals consistently deposit significantly more or less DNA than others [25]. Furthermore, it is interesting to note that, even though volunteers handled the knives on several occasions to simulate regular use, DNA from the regular user of the knives was not always observed as a complete DNA profile. This was the case for volunteer $\mathrm{X}$, presumably because volunteer $\mathrm{X}$ consistently deposited very low amounts of DNA.

\subsection{Detection of indirectly-transferred handshaker DNA}

In contrast to previous experiments involving handshaking and handling of items, in which a major profile matching that of the handshaker was occasionally observed [13, 14], this study found that indirectly-transferred DNA from the handshaker was only observed in three of four pairings of participants, and when it was observed, it was only detected as minor partial profiles. This difference in findings is likely due to the previous studies using pre-cleaned items and a longer hand contact of 1 minute [13, 14]. Longer contact between individuals prior to handling might increase the amount of DNA transferred indirectly and in the absence of a background layer of DNA on the item, DNA deposited during the handling of the item, whether directly or indirectly, would be more easily detected. Here, a shorter handshake was specifically used to be more representative of casual day-to-day contact among people. Knives were set up to have a background layer of regular user DNA present to also be more representative of everyday items, particularly those that are commonly examined in casework, such as, opportunistic weapons. The shorter hand-to-hand contact time 
and background layer of DNA may have therefore resulted in less DNA being transferred and detected from the handshaker.

However, even with these attempts to make the experimental design more representative of real-life scenarios, the possibility of indirect DNA transfer was still maximised to some degree; knives were picked up immediately after handshaking and then stabbed into the foam block for 60 seconds. In order to extend and explore the findings further, it would be of value for this study to be repeated with varying times between handshaking and stabbing, and with shorter durations of stabbing and/or fewer stabbings over the same duration.

\subsection{Detection of indirectly-transferred unknown DNA}

Indirectly-transferred unknown DNA made up less than 4\% of the DNA recovered from the knives of volunteers $\mathrm{W}$ and $\mathrm{Y}$, both before and after the handshaking and stabbing events. A similar level was also observed for volunteer Z's knives before the handshaking and stabbing events, but no indirectly-transferred DNA was detected afterwards. This difference in the detection of non-donor DNA on volunteer Z's knives, although initially surprising, could be due to potential differences in activities of the volunteer prior to handling the knives on different occasions. In these experiments, we did not collect information about the activities of the volunteers just prior to handling the knives; this information may have helped explain the differences observed. Further research is therefore needed to investigate the impact of activities prior to handling an item on the nature and amount of DNA deposited.

Detection of indirectly-transferred unknown DNA alongside expected DNA from the donor has been repeatedly reported, commonly at $\leq 10 \%$ of the profiles obtained, by studies involving handling or wearing of items and DNA profiling using the more sensitive kits [4, 10, 11, 15, 16, 25-28]. It is proposed that hands acquire such nondonor DNA via everyday activities that involve touching other people and other items that have been previously handled $[10,16,20,27,28]$. As such, the recovery of nondonor DNA from handled items is no longer an unexpected observation and should be 
considered a common feature of touch samples [28]. However, Manoli et al [29] detected negligible non-donor DNA on clean plastic tubes that were held by volunteers for 5 minutes, which is in contrast to this and many other studies. Observation of the methods employed though showed that DNA recovery from the tubes was via a single wet swab [29], which has been previously shown to recover less DNA than the more commonly employed double-swab technique of one wet and one dry swab [30] and mini-tapes [31].

A higher proportion of indirectly-transferred unknown alleles were observed on volunteer X's knives, at approximately $25 \%$ before the handshaking and stabbing and $11 \%$ afterwards. These alleles could be attributed to DNA from the volunteer's romantic partner, which supports a previous observation of indirect DNA transfer from a spouse to worn clothing [32]. In addition, the statistical weight attributed to the observation of DNA matching the profile of the volunteer's partner, both through calculations of an RMP and LRs, would be sufficient to use in court, if these mixed profiles were from a crimestain. This emphasises the need to consider the possibility of indirect DNA transfer when interpreting and evaluating mixed DNA profiles in casework that have a known contributor.

\subsection{Persistence of DNA}

The amounts of DNA recovered from the knives after the handshaking and stabbing events did not differ significantly from the amounts of DNA recovered beforehand. This suggests that the DNA deposited during the stabbing of the knives, both directly from the handler and indirectly from the handshaker, was not sufficient to significantly increase the overall total amount of DNA. This could be due to the two-way nature of DNA transfer, such that as DNA was deposited onto the knife handles, DNA was transferred from the knife handles back onto the users' hands. The total quantity of DNA then significantly declined over a week for knives handled by volunteers $W$ and $Z$, but only a slight decline was observed for volunteers $X$ and $Y$, possibly due to these two volunteers depositing the lowest amounts of DNA initially. This decline in DNA quantity was reflected by a significant reduction in peak heights for alleles attributed 
to the regular user of knives for volunteers $\mathrm{W}, \mathrm{X}$ and $\mathrm{Y}$, which also corresponded to a slight decline in the number of alleles observed for $X$ and $Y$. No significant decline in peak heights were observed over the course of the week when volunteer $Z$ was the regular user, possibly due to the greater amounts of DNA deposited by this volunteer.

Consideration of alleles that could be attributed to the respective handshakers showed that indirectly-transferred DNA can persist for at least a week, but was observed only as minor partial profiles [21]. Peak heights of the alleles significantly declined with time for the three volunteer pairings for which handshaker DNA was detected. For pairings $\mathrm{W}: \mathrm{Y}$ and $\mathrm{Y}: \mathrm{W}$, this resulted in a significant decline in profile percentage, as peak heights dropped below the peak height threshold or were not observed at all. In contrast, an apparent increase in profile percentage of handshaker DNA over time was observed for volunteer X's knives. Whilst this might partly be due to stochastic effects encountered with trace samples of DNA, it might also be explained by considering the likely presence of DNA from the volunteer's partner, as seen on the knives prior to the handshaking and stabbing events. For the purpose of this experiment, for the profiles obtained after the handshaking and stabbing events, all alleles that matched the profile of the handshaker and not the regular user were attributed to the handshaker. However, Table 1 shows that there is some overlap between alleles in the handshaker's profile (volunteer Z) and the profile of volunteer X's partner. This likely complicates the interpretation of profiles from volunteer X's knives, as some of the alleles attributed to the handshaker may have come from the partner.

In general, observing a decline in the amounts of DNA recovered and the qualities of the profiles obtained during just one week after deposition is in contrast to that observed by Raymond et al. [9], when considering the persistence of DNA on items within the favourable environmental conditions of the laboratory. However, at $387 \mathrm{ng}$, the initial amount of DNA deposited in that study was notably greater than that present here. In addition, DNA deposition via handling may deposit DNA of poorer quality (given that 'touch DNA' is believed to be comprised of a mixture of nucleated cells, dead cells, and cell-free DNA from sweat) than the cell suspensions and pre-prepared 'naked DNA' used by Raymond et al. [9]. Although, as with that study, investigating 
the effect of varied environmental conditions, such as temperature and humidity, would further increase our understanding of the persistence of 'touch DNA'. Likewise, in this study, DNA persistence was observed on knives that were not exposed to the movements and contacts within packaging or handled further; both of which would likely limit DNA persistence on the handles [33] and should be investigated further.

\subsection{Consideration of the use of empirical data in evaluating DNA evidence}

Being able to attribute DNA to a particular biological source can assist in the evaluation of DNA evidence. For example, in casework, DNA that is attributed to blood or semen can often be associated with the crime in question, whereas it tends to be difficult to do the same with trace DNA, which usually cannot be attributed to a biological source. Recent work into the use of mRNA markers is opening a new avenue to explore regarding attributing trace DNA to skin cells or a particular body fluid (for example, see [20]), which may ultimately assist in inferring the mode of DNA transfer.

In addition to such attribution, it is gaining acceptance that empirical data is required to evaluate DNA evidence at the activity level, but discussion and experiments are needed to establish exactly which aspects of the DNA-specific data should be relied upon. This was recently raised by Samie et al. [5] in terms of which extrinsic characteristics of the DNA profiles obtained in casework may assist in activity level evaluations: DNA concentration (or amount), profile quality (full versus partial profile), or the relative contributions to mixed profiles (major versus minor)? A review of the published literature available in 2013 suggested that the amount of DNA could not be used to infer the mode of DNA transfer [3]. Samie et al. [5] found no clear correlation between DNA concentrations and the quality of DNA profiles obtained from knives that were stabbed into cardboard boxes, which they hypothesised was a consequence of the small quantity of DNA analysed. This suggests that, in certain scenarios, the amount of DNA recovered should not be relied upon to infer mode of transfer, particularly when dealing with trace DNA samples. This is supported by the finding herein that the introduction of indirectly-transferred DNA to knife handles via a handshake did not significantly increase the quantities of DNA recovered. 
With respect to profile quality and the relative contributions to mixed profiles, when specifically considering handled items as is the context of this study, it is clear from the published literature that varied results can be obtained, such that indirectlytransferred DNA can range from not being detected at all to giving a full major profile (for example [15, 16, 27]). It would therefore appear that, under such circumstances and without greater knowledge of associated activities and probabilities, these extrinsic characteristics should also not be used to infer mode of transfer, as has been previously suggested [3]. However, the published studies on which that view was based, along with many of the more recent studies, only consider indirect DNA transfer in the absence of background levels of DNA. First reported by Meakin et al. [21] and expanded herein is the consideration of indirect DNA transfer to items (knife handles) that have a controlled level of background DNA present from prior handling. Under such a situation, indirectly-transferred DNA was only observed as partial minor profiles, suggesting that under specific circumstances, it may be possible to use profile quality and the relative contributions to mixed profiles to distinguish between directlyand indirectly-transferred DNA. Furthermore, persistence of indirectly-transferred DNA was observed for at least a week, although with decreasing numbers of alleles detected, which implies that the sooner an item is sampled after an offence has occurred, the greater the chance of recovering any indirectly-transferred DNA. Whilst these suggestions can be made, this study is an initial investigation into this matter and requires further work to include more volunteers and to consider the effect of a variety of variables, such as, the items handled, the nature and duration of handling (including consideration of activities prior to handling), the nature and duration of contact resulting in indirect transfer, and the period of time between handling and DNA recovery.

\section{Conflicts of interest}

None. 


\section{Acknowledgements}

The authors wish to thank the volunteers for their participation in this study, Fraser Simpson (UCL) for running the samples on the DNA Analyzer, and Dr Denise Syndercombe-Court (King's College London) for providing the UK allele frequencies. Ethical approval for this study was granted by the UCL Research Ethics Committee (Ref. no. 5107/001) and no financial support was received for this work.

\section{References}

[1] R.M. Morgan, J. Flynn, V. Sena, P.A. Bull. Experimental forensic studies of the preservation of pollen in vehicle fires, Science and Justice. 54 (2014) 141-145.

[2] R.A.H. van Oorschot, M.K. Jones. DNA fingerprints from fingerprints, Nature. 387 (1997) 767.

[3] G. Meakin, A. Jamieson. DNA transfer: Review and implications for casework, Forensic Science International: Genetics. 7 (2013) 434-443.

[4] M. Goray, R.A.H. van Oorschot. The complexities of DNA transfer during a social setting, Legal Medicine. 17 (2015) 82-91.

[5] L. Samie, T. Hicks, V. Castella, F. Taroni. Stabbing simulations and DNA transfer, Forensic Science International: Genetics. 22 (2016) 73-80.

[6] R v Reed and Reed, R v Garmson (2009): Court of Appeal of England and Wales (Criminal Division); EWCA Crim 2698, [2010] 1 Cr App R 23.

[7] Fitzgerald v The Queen (2014): HCA 28.

[8] P. Gill. Analysis and implications of the miscarriages of justice of Amanda Knox and Raffaele Sollecito, Forensic Science International: Genetics. 23 (2016) 9-18.

[9] J.J. Raymond, R.A.H. van Oorschot, P.R. Gunn, S.J. Walsh, C. Roux. Trace evidence characteristics of DNA: A preliminary investigation of the persistence of DNA at crime scenes, Forensic Science International: Genetics. 4 (2009) 26-33.

[10] A.E. Fonneløp, H. Johannessen, P. Gill. Persistence and secondary transfer of DNA from previous users of equipment, Forensic Science International: Genetics Supplement Series. 5 (2015) e191-e192.

[11] F. Oldoni, V. Castella, D. Hall. Shedding light on the relative DNA contribution of two persons handling the same object, Forensic Science International: Genetics. 24 (2016) 148-157. 
[12] R.A.H. van Oorschot, G. Glavich, R.J. Mitchell. Persistence of DNA deposited by the original user on objects after subsequent use by a second person, Forensic Science International: Genetics. 8 (2014) 219-225.

[13] R.K. Farmen, R. Jaghø, P. Cortez, E.S. Frøyland. Assessment of individual shedder status and implication for secondary DNA transfer, Forensic Science International: Genetics Supplement Series. 1 (2008) 415-417.

[14] A. Lowe, C. Murray, J. Whitaker, G. Tully, P. Gill. The propensity of individuals to deposit DNA and secondary transfer of low level DNA from individuals to inert surfaces, Forensic Science International. 129 (2002) 25-34.

[15] C.M. Cale, M.E. Earll, K.E. Latham, G.L. Bush. Could secondary DNA transfer falsely place someone at the scene of a crime?, Journal of Forensic Sciences. 61 (2016) 196-203.

[16] C. Davies, J. Thomson, F. Kennedy. Assessing primary, secondary and tertiary DNA transfer using the Promega ESI-17 Fast PCR chemistry, Forensic Science International: Genetics Supplement Series. 5 (2015) e55-e57.

[17] C.M. Cale, K.E. Latham. Authors' Response to Kokshoorn et al, Journal of Forensic Sciences. 61 (2016) 1403-1404.

[18] M. Goray, K.N. Ballantyne, B. Szkuta, R.A.H. van Oorschot. Commentary on Cale CM, Earll ME, Latham KE, Bush GL. Could Secondary DNA Transfer Falsely Place Someone at the Scene of a Crime?, Journal of Forensic Sciences 61 (2016) 13961398.

[19] B. Kokshoorn, B. Aarts, R. Ansell, L. McKenna, E. Connolly, W. Drotz, A.D. Kloosterman. Commentary on Cale CM, Earll ME, Latham KE, Bush GL. Could Secondary DNA Transfer Falsely Place Someone at the Scene of a Crime?, Journal of Forensic Sciences. 61 (2016) 1401-1402.

[20] M. van den Berge, G. Ozcanhan, S. Zijlstra, A. Lindenbergh, T. Sijen. Prevalence of human cell material: DNA and RNA profiling of public and private objects and after activity scenarios, Forensic Science International: Genetics. 21 (2016) 81-89.

[21] G.E. Meakin, E.V. Butcher, R.A.H. van Oorschot, R.M. Morgan. The deposition and persistence of indirectly-transferred DNA on regularly-used knives, Forensic Science International: Genetics Supplement Series. 5 (2015) e498-e500. 
[22] H. Haned, J. de Jong. LRmix Studio 2.0 user manual. (2015) Available online at: http://lrmixstudio.org/wp-content/uploads/2014/12/manual 2.0.pdf

[23] A. Rennison. Guidance: Allele frequency databases and reporting guidance for the DNA (Short Tandem Repeat) profiling, Forensic Science Regulator Codes of Practice and Conduct. FSR-G-213 (2014) Available online at: https://www.gov.uk/government/uploads/system/uploads/attachment data/file/35544 8/FrequencyDatabasesReportingGuidance.pdf.

[24] M. Phipps, S. Petricevic. The tendency of individuals to transfer DNA to handled items, Forensic Science International. 168 (2007) 162-168.

[25] M. Goray, S. Fowler, B. Szkuta, R.A.H. van Oorschot. Shedder status - An analysis of self and non-self DNA in multiple handprints deposited by the same individuals over time, Forensic Science International: Genetics. 23 (2016) 190-196. [26] M. Breathnach, L. Williams, L. McKenna, E. Moore. Probability of detection of DNA deposited by habitual wearer and/or the second individual who touched the garment, Forensic Science International: Genetics. 20 (2016) 53-60.

[27] A.K. Buckingham, M.L. Harvey, R.A.H. van Oorschot. The origin of unknown source DNA from touched objects, Forensic Science International: Genetics. 25 (2016) 26-33.

[28] V.J. Lehmann, R.J. Mitchell, K.N. Ballantyne, R.A.H. van Oorschot. Following the transfer of DNA: How does the presence of background DNA affect the transfer and detection of a target source of DNA?, Forensic Science International: Genetics. 19 (2015) 68-75.

[29] P. Manoli, A. Antoniou, E. Bashiardes, S. Xenophontos, M. Photiades, V. Stribley, M. Mylona, et al. Sex-specific age association with primary DNA transfer, International Journal of Legal Medicine. 130 (2016) 103-112.

[30] B.C.M. Pang, B.K.K. Cheung. Double swab technique for collecting touched evidence, Legal Medicine. 9 (2007) 181-184.

[31] G. Williams, M. Pandre, W. Ahmed, E. Beasley, E. Omelia, D. World, H. Yu. Evaluation of low trace DNA recovery techniques from ridged surfaces, Journal of Forensic Research. 4 (2013).

[32] S.L. Stouder, K.J. Reubush, D.L. Hobson, J.L. Smith. Trace evidence scrapings: A valuable source of DNA?, Forensic Science Communications. 4 (2001). 
[33] M. Goray, R.A.H. van Oorschot, J.R. Mitchell. DNA transfer within forensic exhibit packaging: Potential for DNA loss and relocation, Forensic Science International: Genetics. 6 (2012) 158-166. 